\title{
EMISSÕES PÚBLICAS DE AÇÕES, VOLATILIDADE E INSIDER INFORMATION NA BOVESPA*
}

\section{OTÁVIO RIBEIRO DE MEDEIROS}

Prof. Titular da Universidade de Brasília - DF e do Programa Multiinstitucional de PósGraduação em C. Contábeis UnB, UFPE, UFPB e UFRN

E-mail: otavio@unb.br

\author{
ALBERTO SHIGUERU MATSUMOTO \\ Prof. dos Deptos. de C. Contábeis e \\ Administração da Universidade Católica de \\ Brasília - DF \\ e da Fundação Visconde de Cairu - BA \\ E-mail: albertom@ucb.br
}

\section{RESUMO}

O trabalho utiliza um estudo de evento para examinar os retornos de ações relacionados a emissões públicas por empresas brasileiras listadas na BOVESPA, realizadas entre 1992 e 2002, buscando determinar como o mercado reagiu antes, durante $e$ depois da data do anúncio da emissão. Após utilizar a metodologia convencional de mensuração de retornos anormais por OLS, foram utilizados modelos $\mathrm{ARCH}$ e GARCH, que levam em consideração a heteroscedasticidade condicional da volatilidade dos retornos anormais, em mais de $70 \%$ da amostra, após a constatação da presença desses processos nos resíduos originais. Os resultados mostram que (1) há evidências de insider information antes da data do anúncio, (2) que ocorrem retornos anormais negativos na data do anúncio e (3) que, no período de um ano após as emissões, as ações das empresas que captaram recursos via underwriting tiveram retornos negativos após ajuste ao risco e ao mercado.

Palavras-chave: Estudo de evento, Emissão de ações, Empresas brasileiras, ARCH, GARCH.

\section{ABSTRACT}

The paper uses an event study to examine stock returns associated to public equity issues carried out by Brazilian firms listed on BOVESPA, from 1992 to 2002, with the purpose of determining how the market reacted before, during, and after the issue announcement dates. After utilizing the conventional method for measuring abnormal returns by OLS, we used $A R C H$ and GARCH models, which take into account the conditional heteroskedastic volatility of the abnormal returns in more than $70 \%$ of the sample, since the presence of these processes was found in the original residuals. The results show that (1) there is evidence of insider information prior to the announcement date, (2) abnormal returns occurred on the announcement date, and (3) within one year after the issues, the shares of issuing firms had negative returns after adjusting for risk and market effects.

Keywords: Event Study, Stock Issues, Brazilian Firms, ARCH, GARCH. 


\section{INTRODUÇÃO}

Evidências empíricas sobre as reações do mercado à emissão de ações inspiraram pesquisadores a desenvolver teorias para explicar resultados aparentemente anômalos, contribuindo, assim, para uma melhor compreensão dos mercados de capitais.

Várias hipóteses foram elaboradas para explicar fenômenos de reação do mercado de ações em ofertas públicas. As teorias concorrentes, destinadas a prever os movimentos dos preços das ações em torno das emissões, podem ser agrupadas em três categorias: (a) não há efeito preço: consistente com as hipóteses de mercado eficiente, com as ações sendo consideradas bens substitutos próximos; (b) efeito preço negativo: consistente com efeitos informacionais associados à venda de ações superavaliadas por vendedores bem informados, com hipóteses de estrutura de capital baseadas na redistribuição do valor da empresa entre diferentes categorias de acionistas e com curvas de demanda declinantes por ações; (c) efeito preço positivo: consistente com informações favoráveis associadas aos investimentos e à redução dos custos esperados relacionados a dificuldades financeiras e aos custos de agência.

De acordo com a hipótese de assimetria de informação, uma emissão de ações sinaliza uma superavaliação dos ativos existentes e transmite más notícias sobre o fluxo de caixa futuro da empresa. Por sua vez, as hipóteses de oportunismo da administração afirmam que administradores levantam capital, principalmente, para aumentar o tamanho da empresa e, assim, a sua própria remuneração, o que resultaria em efeitos negativos de preços. A hipótese da curva de demanda declinante supõe que os preços das ações caem quando há anúncio de uma emissão, porque a demanda por ações não é perfeitamente elástica.

O presente trabalho examina os retornos de ações relacionados a 80 emissões públicas de ações por empresas abertas brasileiras ${ }^{1}$ listadas na BOVESPA. Mais especificamente, o estudo investiga, em conexão com essas emissões, realizadas entre 1992 e 2002, se o que ocorre no mercado brasileiro é consistente com as evidências internacionais, no que se refere a: (a) como o mercado reagiu no período que vai de 15 dias úteis antes do anúncio da emissão até a data do anúncio; (b) o que ocorre com o retorno das ações na data do anúncio e dias seguintes e (c) qual o desempenho das ações ao longo de um período de um ano a partir do anúncio da emissão. Inicialmente, o estudo procura mensurar os eventuais retornos anormais das emissões através do modelo de retornos ajustados ao risco e ao mercado (MACKINLAY 1997; SOARES et al., 2002), em que os retornos anormais são os resíduos de uma regressão linear simples (OLS). No entanto, conforme evidenciado empiricamente (BOLLERSLEV, 1986; AKGIRAY, 1989; CHIANG e DOONG, 2001), é comum que os retornos das ações sigam processos auto-regressivos com inovações (resíduos) condicionalmente heteroscedásticas, isto é, padrões ARCH ou GARCH ${ }^{2}$. De fato, processos desse tipo foram detectados no presente trabalho em mais de $70 \%$ da amostra. Para esses casos, a estimação original por OLS foi substituída pela estimação por ARCH ou GARCH, conforme necessário, com uma qualidade de ajustamento muito superior àquela, sendo os retornos anormais obtidos dessa forma. O restante do trabalho está delineado da seguinte forma: a Seção 2 resume teorias e estudos empíricos anteriores; a Seção 3 discorre sobre a amostra e as metodologias utilizadas; a Seção 4 analisa os resultados e a Seção 5 apresenta as conclusões.

\section{TEORIAS E EVIDÊNCIAS EMPÍRICAS}

Há diversos enfoques teóricos que procuram explicar a reação dos mercados ao anúncio de emissões públicas de ações. Estão sintetizadas a seguir as teorias mais relevantes sobre o assunto.

Myers e Majluf (1984) estudaram o funding das corporações e as decisões de investimento sob a suposição de que os administradores possuem meIhor informação sobre o valor da empresa vis-à-vis os acionistas externos. Mostraram que, no melhor interesse dos acionistas existentes, os administradores podem rejeitar racionalmente projetos com VPL - Valor Presente Líquido positivo, pois, se as ações da empresa estão subavaliadas pelo mercado, a diluição sofrida pelos acionistas existentes em conseqüência a uma emissão pode ser maior que o ganho que eles teriam com a empresa investindo em projetos com VPL positivo. Assim, os administradores evitarão as emissões de ações para projetos que necessitam de financiamento através de capital próprio. Por outro lado, uma decisão de emitir

\footnotetext{
' O presente trabalho trata apenas de emissões públicas não iniciais de ações, conhecidas na literatura internacional como SEOs (Seasoned Equity Offerings). Portanto, quando o texto menciona emissões de ações, estará implícito que se refere apenas a esse tipo de emissão, excluindo-se, portanto, as emissões de ações associadas a aberturas de capital, subscrições privadas, bonificações, emissão de ADRs etc.

${ }^{2}$ ARCH - Autoregressive Conditionally Heteroscedastic; GARCH - Generalized Autoregressive Conditionally Heteroscedastic.
} 
ações para investir em projetos, pode sinalizar uma superavaliação das ações da empresa. Essa sub/ superavaliação das ações gera um problema de seleção adversa: por um lado, a decisão teoricamente correta (investir em projetos com VPL positivo) pode ser pior para os acionistas; por outro, investidores podem achar que se a empresa precisa de recursos, então seu fluxo de caixa futuro não é bom, logo as ações estão superavaliadas. Assim, anúncios de emissão de ações podem resultar num impacto negativo no preço da ação, explicando, assim, retornos anormais negativos.

Miller e Rock (1985) assumem que os administradores conhecem melhor o fluxo de caixa futuro do que os acionistas, mas que não há tal assimetria informacional sobre o nível de investimento planejado e sobre o valor dos ativos condicionados ao fluxo de caixa presente. Nesse modelo, uma inesperada decisão de emitir ações sinaliza más notícias sobre a capacidade de o fluxo de caixa futuro financiar os investimentos planejados, o que explicaria uma resposta negativa em termos de preço da ação.

O problema de seleção adversa e o timing de novas emissões foram o objeto da hipótese de sinalização de Lucas e McDonald (1990) em seu modelo de informação assimétrica sobre a decisão de emissão de ações, o qual prevê que as emissões são, em média, precedidas por um retorno anormal positivo da ação e que o preço da ação cai quando do anúncio de uma emissão.

Jensen e Meckling (1976) relacionaram o custo de agência às novas emissões de ações. Nos Estados Unidos, boa parte da remuneração dos executivos é feita sob a forma de ações ou opções de compra de ações. Assim, no processo decisório de emitir ações, custos de agência surgiriam da divergência entre 0 interesse dos administradores e o dos acionistas. Jensen (1986), também, recorre à teoria da agência para prever reações do mercado às emissões de ações. Administradores são os agentes dos acionistas. Uma vez que ambas as partes têm interesses particulares, podem ocorrer sérios conflitos entre eles quanto à escolha da meIhor estratégia corporativa. A reação do mercado ao anúncio de uma emissão de ações dependerá da avaliação do mercado de que os recursos captados serão investidos em projetos com valor presente líquido positivo ou não.

Scholes (1972) propôs que os preços das ações podem cair quando do anúncio de uma emissão de ações porque a curva de demanda em relação a essas ações é declinante. O modelo contraria a hipótese de mercado eficiente em que a curva de demanda pelas ações é horizontal e as ações são bens substitutos próximos. A hipótese de Scholes supõe um mercado de capitais incompleto com vendas a descoberto (short-sales). Nessas condições, não existiriam substitutos perfeitos para as ações de uma empresa no mercado. Na ausência de substitutos perfeitos, as empresas se defrontarão com curvas de demanda declinantes por suas ações. A hipótese de Scholes prevê que um aumento na quantidade de ações, causada por uma nova emissão, resultará numa queda permanente no preço da ação.

Atualmente, a maior parte da evidência empírica existente é suportada pelo fato de que o mercado reage, negativamente, aos anúncios de emissões de ações. No entanto, Hess e Frost (1982) investigaram movimentos de preço nas imediações da data de emissão de SEOs (Seazoned Equity Offers), isto é emissões públicas não-iniciais de ações, comparando três hipóteses concorrentes: a visão da SEC - Securities Exchange Comission de que uma nova emissão causa uma queda permanente de preço; a visão dos underwriters de que há apenas uma queda temporária de preço durante o período de distribuição e a hipótese de mercado eficiente de que não há efeito de preços. Os autores concluem que as duas primeiras hipóteses devem ser rejeitadas em favor da hipótese de mercado eficiente.

Ao contrário, Asquith e Mullins (1986) mostraram que anúncios de emissões de ações provocam quedas significativas no preço das ações, ao encontrarem retornos médios anormais de $-3 \%$ no período de dois dias após os anúncios de emissões. Essa reação negativa de preço, representando uma perda no valor das empresas, corresponderia em média a $31 \%$ dos recursos captados nas emissões. Essa perda é o que normalmente se chama de diluição. A constatação é consistente tanto com a hipótese de sinalização, em que as emissões de ações transmitem informação negativa aos investidores sobre o verdadeiro valor da empresa, bem como a hipótese da pressão sobre os preços de que há uma curva de demanda declinante pelas ações.

Uma análise realizada por Mikkelson e Partch (1986) concluiu que as emissões realizadas estariam associadas a um retorno extraordinário médio positivo na data do anúncio e a um retorno médio negativo na data de emissão. O motivo declarado para a emissão também afetaria a reação de preço. O estudo documenta uma queda maior de preço em resposta a emissões de ações ordinárias para refinanciar dívida do que para financiar investimentos de capital. Essa evidência é consistente com Myers e Majluf (1984) quanto ao argumento de que anúncios de emissões de ações ordinárias e de debêntures conversíveis sinalizam que o preço das ações está muito alto. Respostas negativas de preço foram 
documentadas para todos os tipos de emissões novas não esperadas, o que está de acordo com o modelo de Miller e Rock (1985) de que tais decisões levam os participantes do mercado a rebaixar a sua avaliação quanto às perspectivas de lucros futuros da empresa.

Gerard e Nanda (1993) desenvolveram um modelo que prevê, inter alia, que os preços das ações no mercado secundário caem nos dias que precedem a emissão, e recuperam-se após a emissão, e que as novas ações são vendidas com deságio com relação à expectativa do mercado quanto ao valor da ação na data de emissão.

Loughran e Ritter (1995) mostraram que as ações das empresas que emitiram entre 1970 e 1990, sejam emissões iniciais ou não, tiveram um desempenho significativamente inferior às empresas que não emitiram durante cinco anos após as respectivas datas de emissão. Os autores evidenciaram, também, que as empresas emitentes têm betas ligeiramente maiores do que as não emitentes, indicando que as emitentes deveriam ter retornos mais altos e não mais baixos que as demais. Nas palavras dos autores, isso constitui um enigma (puzzle): por que as empresas que emitem ações geram retornos tão baixos para os investidores nos cinco anos subseqüentes à emissão?

Spiess e Affleck-Graves (1995) e Loughran e Ritter (1997), também, mostraram que, além dos retornos negativos quando dos anúncios, empresas emitentes sofrem retornos anormalmente baixos nos cinco anos subseqüentes às emissões. Uma explicação para isso é que os administradores datam as emissões para tirar proveito de "janelas" de oportunidade para emitir ações superavaliadas. Essa explicação requer não somente que os investidores sejam excessivamente otimistas sobre as perspectivas de lucros das empresas emitentes, mas também que os investidores sub-reajam às informações transmitidas pela emissão. Embora Fama (1998) tenha argumentado que esses resultados refletem variações aleatórias normais que podem ocorrer em mercados eficientes, o desempenho anormal de longo prazo no preço das ações constitui-se num desafio ao tradicional paradigma de eficiência de mercado.

Dois modelos comportamentais conflitantes, de sub-reação e super-reação, respectivamente, foram desenvolvidos por Kadiyala e Rau (2001) para explicar os fenômenos associados a retornos anormais de longo prazo subseqüentes às emissões. $O$ estudo forneceu evidência de que retornos anormais negativos só podem ser atribuídos ao modelo de sub-reação do investidor. Investidores sub-reagiriam à informação de curto prazo disponível an- tes do evento e, subseqüentemente, à informação transmitida pelo evento.

Hertzel et al. (2002) examinaram o desempenho pós-anúncio do preço das ações para uma amostra de empresas que realizam emissões privadas. Os autores descobriram que apesar da reação de preço positiva no anúncio, as empresas que emitem ações privadamente têm um desempenho acionário inferior a vários benchmarks ao longo de três anos após a emissão. O desempenho negativo pós-anúncio, documentado para as emissões privadas, é similar ao baixo desempenho de longo prazo documentado para emissões públicas iniciais (IPOs) ou não iniciais (SEOs). Em conjunto, a evidência sugere que os investidores são demasiadamente otimistas sobre as perspectivas das empresas emitentes, independentemente da forma de emissão.

Leal e Amaral (2000) estudaram a reação do mercado brasileiro antes e no anúncio de uma amostra de emissões públicas, tendo constatado retornos anormais positivos a 60 dias e a 5 dias antes do anúncio, denotando uma possível manipulação por parte de investidores com insider information. Constataram, ainda, reações negativas do mercado na data do anúncio, em consonância com os estudos norte-americanos citados.

\section{METODOLOGIA}

No período 1992-2002, foram realizadas 172 emissões públicas não-iniciais de ações. Desse total, foram excluídas aquelas cujas ações têm baixa liquidez, impedindo a realização de regressões com um número adequado de observações. Das emissões restantes, 80 foram selecionadas aleatoriamente. Assim, no presente estudo, 80 emissões realizadas por empresas com ações incluídas no IBOVESPA foram selecionadas. Essas emissões estão listadas no Anexo, por empresa e datas de anúncio, ao final do artigo. A fonte dos dados foi a Economática ${ }^{\circledR}$. As emissões ocorreram entre 1992 e 2002. A janela de evento inclui o dia do anúncio da emissão e os dois dias úteis subseqüentes. $O$ estudo analisa, também, os retornos anormais observados no período que se inicia 15 dias úteis antes da data do anúncio da emissão e termina um ano após a data de emissão. A análise dos retornos nos 15 dias que antecedem o anúncio tem por propósito verificar se há indícios de informação privilegiada (insider information), o que produziria retornos anormais antes da data do anúncio. A análise dos retornos no período de um ano após o anúncio teve por objetivo verificar se a evidência empírica de que o desempenho das ações de empresas emitentes, também, é negativo no Brasil, no longo prazo. 
Visando verificar a estabilidade das séries, foi aplicado nas 160 séries de dados da amostra, isto é, nas 80 séries de retornos das ações e nas 80 séries dos correspondentes retornos do mercado, o teste ampliado de Dickey-Fuller para raízes unitárias (DICKEY e FULLER, 1979), de modo a prevenir a ocorrência de regressões espúrias, em caso de existência de tais raízes. A literatura indica que os preços das ações tendem a ser não-estacionários, enquanto que os retornos das ações tendem a ser estacionários (BROOKS, 2002). Os resultados estão descritos na seção seguinte.

A metodologia utilizada no presente estudo teve como objetivo verificar:

(a) se há retornos anormais no período de 15 dias úteis anteriores ao anúncio, o que indicaria a ocorrência de insider information;

(b) se há retornos anormais negativos quando do anúncio da emissão, conforme documentado em estudos empíricos anteriores e

(c) se o desempenho das ações das empresas emitentes, no Brasil, é também negativo no longo prazo, conforme a evidência internacional.

Existem dois tipos de modelos para o estudo empírico do comportamento das ações nos mercados de capitais: modelos de preços e modelos de retorno. ${ }^{3}$ Devido ao fato de que modelos de preços apresentam problemas econométricos mais complexos, os modelos de retorno são utilizados com maior freqüência. No presente trabalho, um determinado tipo de modelo de retorno é utilizado no contexto da metodologia conhecida na literatura como estudo de evento. Fundamentos sobre o tema podem ser encontrados, por exemplo, em Brown e Warner (1985), Campbell, Lo e MacKinlay (1997), MacKinlay (1997) e Soares et al. (2002).

No estudo, cada evento é definido como o anúncio de uma emissão por uma empresa listada $e$ a data do evento é a data do anúncio da emissão. A data de anúncio foi definida como aquela em que a primeira informação sobre a emissão tornou-se pública. As fontes de informação para a definição da data de anúncio para cada emissão foram jornais especializados em economia e finanças onde são normalmente publicados tais anúncios, normalmente "Gazeta Mercantil" ou "Valor Econômico", na forma de fato relevante, aviso aos acionistas, edital de convocação de Assembléia Extraordinária etc.
O estudo utiliza retornos diários, considerando-se apenas os dias úteis. Os testes de hipóteses são realizados para a hipótese $\mathrm{H}_{0}$ de não existência de retornos anormais. Com o objetivo de capturar possíveis retornos anormais na janela de evento, utilizou-se, inicialmente, o modelo de mercado, ou modelo de retorno ajustado ao risco e ao mercado, estimado por OLS, conforme descrito a seguir.

$\mathrm{Na}$ data em que as ações da empresa passaram de "negociadas com-direito" (de subscrição) para "ex-direito", foi realizado o seguinte ajuste no cálculo do retorno daquele dia: $\mathrm{PEx}=(\mathrm{PA}+\mathrm{PS} \times$ $\mathrm{Pr}) /(1+\mathrm{Pr})$, em que PEx é o preço ex-direito, PA é o preço anterior, com-direito, PS é o preço de subscrição e Pr é a proporção do aumento de capital a que $o$ acionista tem direito a subscrever.

\subsection{Mensuração dos Retornos Anormais por OLS}

Existem diversos métodos alternativos para se calcular os retornos anormais, seja por meio de modelos estatísticos ou econômicos ${ }^{4}$. No presente estudo, o modelo de mercado 5 foi, inicialmente, utilizado, de modo que os retornos anormais para cada evento foram obtidos através da diferença entre os retornos observados e os retornos ajustados, esses baseados na estimação por mínimos quadrados (OLS), tendo como variável explanatória o retorno de mercado. Isso pode ser expresso por:

$$
A R=R-R^{*},
$$

em que AR é o vetor coluna dos retornos anormais, $R$ o vetor coluna dos retornos observados e $\mathrm{R}^{*}$ é vetor coluna dos retornos ajustados. A equação de regressão utilizada para estimar os parâmetros é dada por:

$$
R=R_{m} \beta+u,
$$

em que $R_{m}$ é uma matriz com os elementos da $1^{a}$ coluna iguais à unidade e a $2^{\mathrm{a}}$ coluna formada pelos retornos de mercado ao longo do tempo. $\beta$ é um vetor coluna com os parâmetros da regressão, e u o vetor coluna de termos aleatórios $u_{t}$ especificados como i. i. d. $\sim \mathrm{N}\left(0, \sigma^{2}\right)$. O retorno ajustado é dado por:

$$
\begin{aligned}
& R^{*}=R_{m} \hat{\beta}, \text { em que } \\
& \hat{\beta}=\left(R_{m}^{\prime} R_{m}\right)^{-1} R_{m}^{\prime} R,
\end{aligned}
$$

\footnotetext{
${ }^{3}$ Vide Kothari e Zimmerman (1995).

${ }^{4}$ Para uma descrição detalhada vide McKinley (1997) ou Brown e Warner (1985).

${ }^{5}$ Também denominado de modelo de retorno ajustado ao risco e ao retorno (SOARES et al., 2000).
} 
sendo $\hat{\beta}$ o vetor dos parâmetros estimados da regressão linear, para uma janela de estimação anterior ao evento, e $\mathrm{R}_{\mathrm{m}}$ é a matriz dos retornos de mercado, cuja proxy é o retorno do índice BOVESPA. Nesse estudo, a janela de estimação adotada para todos os eventos da amostra foi de 105 dias. Assumindo que os elementos de $R_{m}$ são exógenos com relação a $\mathrm{R}$, então os parâmetros de $\hat{\beta}$ são estimativas não enviesadas e consistentes. Combinando (2) e (3) resulta:

$$
A R=R-R_{m} \hat{\beta} .
$$

A agregação dos retornos anormais através da amostra de tamanho $N$ é dada por:

$$
\overline{A R}=\frac{1}{N} \sum_{i=1}^{N} A R \text {. }
$$

Os retornos anormais médios diários obtidos em (6) devem ser acumulados ao longo do tempo. Definindo $\overline{\operatorname{CAR}}\left(\tau_{1}, \tau_{2}\right)$ como os componentes do vetor coluna dos retornos médios anormais cumulativos entre as datas $\tau_{1}$ e $\tau_{2}$, então:

$$
\overline{C A R}=\left(\tau_{1}, \tau_{2}\right)=\sum_{\tau_{1}}^{\tau_{2}} \overline{A R_{t}} .
$$

Sob a hipótese nula de que $\overline{\operatorname{CAR}}\left(\tau_{1}, \tau_{2}\right)=0$, temse:

$$
\theta=\frac{\overline{C A R}\left(\tau_{1}, \tau_{2}\right)}{\left[\operatorname{var} \overline{(C A R}\left(\tau_{1}, \tau_{2}\right)\right]^{1 / 2}}: N(0,1) .{ }^{6}
$$

\subsection{Mensuração dos Retornos Anormais por ARCH/GARCH}

Bollerslev (1986), Akgiray (1989) e Chiang e Doong (2001) mostraram que os retornos diários das ações têm volatilidade variável ao longo do tempo e tendem, assim, a apresentar efeitos ARCH/GAR$\mathrm{CH}$. Decidiu-se, no presente estudo, considerar a possibilidade dessa ocorrência, através do teste de Engle (1982). De fato, em 58 dos 80 eventos componentes da amostra, verificou-se que a hipótese nula de não existência de efeitos $\mathrm{ARCH}(1)$ ou GAR$\mathrm{CH}(1,1)$ foi fortemente rejeitada. Dos 58 casos, 13 casos foram processos $\mathrm{ARCH}(1)$ e 45 foram processos GARCH(1,1). Nessa situação, a estimação por OLS é espúria, pois a premissa de resíduos i. i. d $\mathrm{N}\left(0, \sigma^{2}\right)$ é violada. Por esse motivo, ao se detectar a presença daqueles processos, as regressões repre- sentadas por (2) foram re-estimadas utilizando-se os modelos GARCH ou ARCH, conforme necessário. Na especificação $\mathrm{GARCH}(1,1)$, a especificação (2) é substituída por:

$$
\begin{gathered}
R=R_{m}+u \quad u_{i t} \sim N\left(0, \sigma_{i t}^{2}\right), \text { em que } \\
\sigma_{i t}^{2}=\alpha_{i 0}+\alpha_{i 1} u_{i t-1}^{2}+\alpha_{i 2} \sigma_{i t-1}^{2} .
\end{gathered}
$$

Essa especificação reduz-se a um processo $\mathrm{ARCH}(1)$ se $\alpha_{\mathrm{i} 2}=0$.

O conjunto de métodos, utilizados no estudo, incluiu ainda o teste de White (1980) de heteroscedasticidade, o teste de Jarque-Bera de normalidade dos resíduos (BERA; JARQUE, 1981), o teste de Durbin-Watson de autocorrelação dos resíduos (DURBIN; WATSON, 1951). A estimação pelo método de Cochrane-Orcutt (COCHRANE; ORCUTT, 1949), foi utilizada quando da ocorrência de autocorrelação nos resíduos OLS.

\section{RESULTADOS}

Conforme esperado, o teste ampliado de DickeyFuller resultou na rejeição de $\mathrm{H}_{0}$ quanto à presença de raízes unitárias em todas as séries, ao nível de $1 \%$, de forma que as séries foram consideradas estacionárias ou integradas I(0). Um aspecto negativo é que o teste de normalidade de Jarque-Bera a $5 \%$ revelou que em 39 das 80 regressões a hipótese $\mathrm{H}_{0}$ de normalidade dos resíduos é aceita, sendo rejeitada para as demais. A normalidade dos resíduos é necessária para a validade dos testes $t$. Felizmente, tendo em vista que em metade da amostra os resíduos mostraram-se gaussianos e ao fato de que a amostra é suficientemente grande $(N=80)$, a violação da normalidade dos resíduos na outra metade da amostra é praticamente inócua, dado o Teorema do Limite Central. ${ }^{7}$

Quanto à mensuração dos retornos anormais por OLS, conforme a equação (2), o teste de Durbin-Watson revelou que em 3 regressões a hipótese nula de não existência de correlação serial nos resíduos foi rejeitada. Para essas regressões, utilizouse o método de Cochrane-Orcutt para a estimação, eliminando o problema de autocorrelação.

Tendo em vista a possibilidade de existência de efeitos ARCH ou GARCH nos resíduos das regressões por OLS, decidiu-se aplicar o teste de Engle (1982) nas 80 regressões, tendo-se verificado a ocorrência desses efeitos em 58 regressões. Por esse motivo, decidiu-se re-estimar essas regressões utili-

\footnotetext{
${ }^{6}$ Tendo em vista que a variância dos retornos é desconhecida, o teste z reduz-se a um teste $\mathrm{t}$ com $\mathrm{N}-1=79$ graus de liberdade
}

${ }^{7}$ Vide Brooks (2002, p. 182). 
zando os modelos ARCH(1) ou GARCH(1,1), de acordo com as equações (9) e (10) acima. A ocorrência de GARCH $(1,1)$ mostrou-se significativa em 45 casos e a de $\mathrm{ARCH}(1)$ em 13 casos, sempre abaixo de $5 \%$ de significância. Para esses casos, as regressões originais por OLS foram, então, substituídas pelos modelos ARCH ou GARCH, mantendo-se as regressões originais por OLS para as 22 restantes. As regressões obtidas por ARCH ou GARCH mostraram-se significativamente superiores àquelas estimadas por OLS, tanto em termos de teste $t$ dos parâmetros, do teste $\mathrm{F}$ e do $\mathrm{R}^{2}$. O teste de White não revelou a existência de outros processos heteroscedásticos além daqueles do tipo ARCH e GARCH acima citados.
O Gráfico 1 mostra duas séries: (a) os resultados obtidos com estimação por OLS para toda a amostra e (b) com por ARCH para 13 eventos, GAR$\mathrm{CH}$ para 45 eventos e OLS para 22 eventos. Pode-se verificar que os resultados da estimação unicamente por OLS levam a uma série de retornos anormais mais baixos que a série estimada pela combinação de OLS, ARCH ou GARCH, indicando que os resultados estimados exclusivamente por OLS são enviesados para baixo. Verifica-se, no entanto, que a re-estimação de parte da amostra por $\mathrm{ARCH}$ ou GARCH não altera qualitativamente as conclusões com relação à reação do mercado.

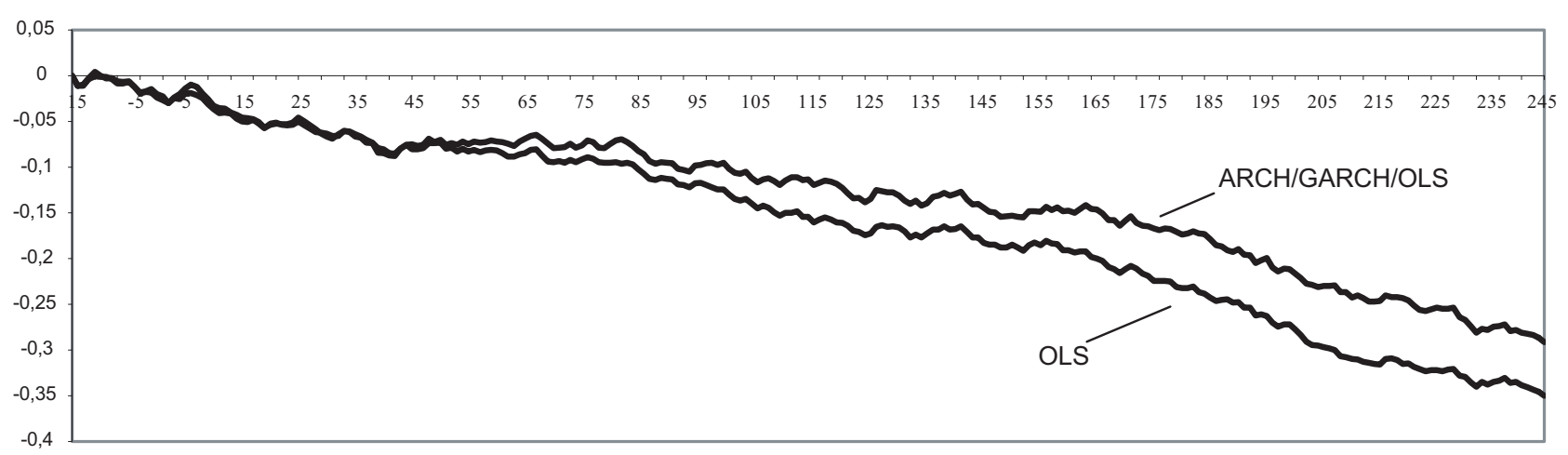

GRÁFICO 1 - RETORNOS ANORMAIS ACUMULADOS

\begin{tabular}{|c|c|c|c|c|c|c|c|}
\hline dia & AR & CAR & t(CAR) & dia & AR & CAR & t(CAR) \\
\hline-14 & $-0,011$ & $-0,011$ & $-3.011^{*}$ & +1 & $-0,003$ & $-0,027$ & $-1.833^{*}$ \\
\hline-13 & 0,000 & $-0,011$ & $-2.080^{*}$ & +2 & $-0,003$ & $-0,030$ & $-1.986^{\star}$ \\
\hline-12 & 0,007 & $-0,004$ & $-0,624$ & +3 & 0,006 & $-0,024$ & -1.523 \\
\hline-11 & 0,003 & $-0,001$ & $-0,077$ & +4 & 0,003 & $-0,020$ & -1.278 \\
\hline-10 & $-0,001$ & $-0,001$ & $-0,160$ & +5 & 0,006 & $-0,014$ & $-0,857$ \\
\hline-9 & 0,000 & $-0,001$ & $-0,133$ & +6 & 0,004 & $-0,010$ & $-0,588$ \\
\hline-8 & $-0,003$ & $-0,004$ & $-0,406$ & +7 & $-0,003$ & $-0,012$ & $-0,727$ \\
\hline-7 & $-0,004$ & $-0,008$ & $-0,799$ & +8 & $-0,007$ & $-0,020$ & -1.119 \\
\hline-6 & 0,000 & $-0,008$ & $-0,775$ & +9 & $-0,006$ & $-0,026$ & -1.427 \\
\hline-5 & 0,002 & $-0,007$ & $-0,589$ & +10 & $-0,007$ & $-0,033$ & $-1.797^{*}$ \\
\hline-4 & $-0,005$ & $-0,012$ & $-0,988$ & +11 & $-0,003$ & $-0,036$ & $-1.911^{*}$ \\
\hline-3 & $-0,008$ & $-0,020$ & -1.567 & +12 & 0,000 & $-0,036$ & $-1.885^{\star}$ \\
\hline-2 & 0,002 & $-0,017$ & -1.325 & +13 & $-0,004$ & $-0,040$ & $-2.084^{*}$ \\
\hline-1 & $-0,001$ & $-0,018$ & -1.333 & +14 & $-0,003$ & $-0,043$ & $-2.185^{\star}$ \\
\hline 0 & $-0,006$ & $-0,024$ & $-1.681^{*}$ & +15 & $-0,003$ & $-0,046$ & $-2.293^{\star}$ \\
\hline * Significativo & $-5 \%$. & & & & & \\
\hline
\end{tabular}

Quadro 1 - Retornos anormais (AR), retornos anormais acumulados (CAR) e estatísticas $t$, estimados por ARCH e GARCH e OLS 
O Quadro 1 mostra os retornos anormais diários simples e acumulados e estatísticas t estimados por OLS, ARCH ou GARCH, de acordo com o melhor diagnóstico, para o período de 15 dias antes e 15 dias após o anúncio da emissão. Observa-se a ocorrência de retornos anormais negativos significativos nos $14^{\circ}$ e $13^{\circ}$ dias anteriores ao anúncio, seguidos de retornos não significativos. No dia do anúncio e nos dois dias subseqüentes, há também retornos anormais negativos significativos. Entre o $3^{\circ}$ e o $9^{\circ}$ dia após o anúncio, não há retornos significativos, mas a partir do $10^{\circ}$ dia, os retornos anormais negativos são significativos até o final do período de aproximadamente um ano (em dias úteis).

Com base nesses resultados empíricos, pode-se dizer que:

(a) a reação do mercado às emissões no período pré-anúncio mostram um retorno anormal negativo 14 dias úteis antes do anúncio (3 semanas), parecendo indicar a ocorrência de insider information, antecipando a reação que deveria ocorrer na data do anúncio;

(b) há retornos anormais cumulativos negativos na data do anúncio e nos dois dias subseqüentes, conforme as evidências anteriores e

(c) a reação do mercado ao longo de aproximadamente um ano após o anúncio das emissões indica um desempenho negativo das ações, conforme as evidências anteriores.

Confrontando esses resultados com as discussões apresentadas nas Seções 2 e 3, pode-se dizer que os resultados são consistentes com as bases teórica e empírica existentes. Os retornos anormais negativos no anúncio são consistentes com a hipótese de informação assimétrica na qual a administração possui melhor informação sobre o valor da empresa do que os investidores externos. Mais especificamente, os resultados estão de acordo com as hipóteses de Myers e Majluf (1984), e Miller e Rock (1985) de que a emissão transmite informação negativa sobre as perspectivas futuras das empresas. Os resultados do trabalho não contradizem inteiramente a hipótese de sinalização de Lucas e McDonald (1990). Embora aquele modelo preveja que emissões de ações são em média precedidas por um retorno anormal positivo da ação, o que não se observou no atual estudo, ele também permite quedas de preço relativas ao mercado, i. e., retornos anormais negativos após o anúncio emissão.

A ocorrência de retornos anormais negativos a três semanas antes do anúncio pode ser interpreta- da como indícios de insider information, pois parece haver uma antecipação do que deveria ocorrer apenas na data do anúncio. No entanto, esse resultado é contrário ao obtido em Leal e Amaral (2000), no que tange ao período pré-anúncio, pois eles encontraram retornos anormais positivos a 60 e a 5 dias do anúncio, indicando manipulação para obtenção de ganhos por parte de insiders. O resultado do presente estudo com relação à data do anúncio, entretanto, está de acordo com Leal e Amaral (2000).

Os resultados apresentados são também compatíveis com Scholes (1972), Jensen e Meckling (1976), Asquith e Mullins (1986), Myers e Majluf (1984) Miller e Rock (1985), Spiess e Affleck-Graves (1995) e Loughran e Ritter (1995), pois todos esses estudos relatam retornos anormais negativos no anúncio das emissões e/ou no longo prazo, após as emissões. Os números aqui obtidos não confirmam as previsões do modelo de Gerard e Nanda (1993), no qual na média os preços no mercado secundário caem nos dias que antecedem a data de emissão e recuperam-se após a emissão, pois tal recuperação não se verificou. Ao serem comparados com os de Hess e Frost (1982), os resultados também não confirmam a conclusão desses autores de que as visões da SEC (queda permanente preço) e do underwriter (queda temporária de preço) devem ser rejeitadas em favor da hipótese de mercado eficiente (não há efeito preço).

\section{CONCLUSÕES}

O presente trabalho apresentou um estudo de evento sobre a reação de mercado observada em relação a uma amostra de 80 emissões de empresas realizadas na BOVESPA entre 1992 e 2002. Como foi mencionado, o artigo trata de emissões públicas e, portanto, não estão contemplados aumentos de capital por incorporação de reservas, emissões de ADR ou particulares, aberturas de capital etc. Inicialmente, os retornos anormais foram calculados para cada emissão utilizando-se o modelo de mercado, com estimação por OLS. Verificou-se, entretanto, a presença de processos ARCH ou GARCH nos resíduos das regressões, em $72 \%$ dos casos. Para esses casos, as regressões foram re-estimadas por modelos ARCH ou GARCH, buscando-se o melhor ajustamento possível aos dados. Comparando-se os resultados iniciais com aqueles que incluem os processos condicionalmente heteroscedásticos, verificou-se que os primeiros indicam retornos anormais negativos enviesados para baixo em relação à estimação alternativa. A re-estimação, entretanto, embora leve a retornos anormais menores, em valor absoluto, não alteram qualitativamente os resultados. 
Assim, os resultados mostraram que os acionistas parecem ficar céticos quando as empresas vão ao mercado buscar recursos, com o argumento de que estão investindo em projetos com VPL positivos que poderiam maximizar a riqueza de seus proprietários. Talvez seja verdade a prazo superior a um ano, o que não foi objeto deste estudo.

Quando as empresas anunciam a captação de recursos via emissão de novas ações, poderia se inferir que elas estavam esperando a janela (de oportunidade) de mercado se abrir. Assim, quando a BOVESPA atinge, depois de seguidas altas, um determinado nível em que as ações dessas empresas ficam superavaliadas, os proprietários ficam satisfeitos em vender parte de seu investimento com lucro ou quando o valor de mercado do investimento encontra-se com um determinado preço em que não deverá ser atingido pelo fluxo de caixa futuro descontado a valor presente, dos próximos anos. Há, dessa forma, uma sinalização negativa ao mercado quando as empresas anunciam a captação de recursos, via subscrição pública de ações, pois se supõe que os administradores conhecem melhor o fluxo de caixa do que os acionistas minoritários, em consonância com os estudos de Myers e Majluf (1984), e Miller e Rock (1985)

Conforme demonstrado por este estudo, no período de 1992 a 2002, as empresas, na média, que captaram recursos via underwriting, não conseguiram igualar o benchmark, utilizado, ou seja, o
Índice Bovespa. Pode-se inferir que há aqueles que, tendo conhecimento da subscrição pública antes do mercado (insiders information), vendem sua posição acionária a cerca de três semanas antes do anúncio, tendo em vista que o anúncio transmitirá informação negativa sobre o verdadeiro valor da empresa. A conclusão geral é de que há retornos anormais negativos significativos cerca de 3 semanas antes do anúncio, indicando a ocorrência de inside information, que anteciparia o que deveria ocorrer somente na data do anúncio.

Os resultados apresentaram um CAR negativo de 4,6\% nos 30 dias ao redor da data do anúncio, ou seja, 14 dias antes e 15 dias depois. Na data do anúncio houve um retorno anormal (AR) negativo de $0,6 \%$ na média das ações pesquisadas, significativo ao nível de $5 \%$. Pode-se afirmar que os resultados obtidos são consistentes com a maior parte da evidência empírica prévia, tanto em termos dos retornos anormais negativos no anúncio, bem como ao longo dos 12 meses seguintes. Os resultados parecem mostrar que a reação do mercado às emissões públicas não iniciais de ações no Brasil não é muito diferente daquela observada nos Estados Unidos, pelo menos quando o período de um ano é considerado. Deve-se mencionar, porém, que a amostra aqui utilizada é relativamente pequena, embora tenham sido pesquisados 11 anos, em comparação com aquelas relatadas em estudos realizados nos mercados de capitais daquele país.

\section{REFERÊNCIAS BIBLIOGRÁFICAS}

AKGIRAY, V.. Conditional Heteroscedasticity in Time Series of Stock Returns: Evidence and Forecasts. Journal of Business, vol. 62 , n. 1, p. 55-80, 1989.

ASQUITH, P.; MULLINS, D. W. Jr.. Equity Issues and Offering Dilution. Journal of Financial Economics 15, p. 61-89, 1986.

BERA, A. K.; JARQUE, C. M.. An Efficient Large-Sample Test for Normality of Observations and Regression Residuals. Australian National University Working Papers in Econometrics n. 40, Canberra, 1981.

BOLLERSLEV, T.. Generalized Autoregressive Conditional Heteroscedasticity, Journal of Econometrics n. 31, p. 307-327, 1986.

BROOKS, C.. Introductory Econometrics for Finance. Cambridge, UK: Cambridge U. Press, 2002.

BROWN, S. J.; WARNER, J. B.. Using daily stock returns: the case of event studies. Journal of Financial Economics n. 14, 1985 p. 3-31, 1985.

CAMPBELL, J. Y.; LO, A. W.; MACKINLEY, A. C.. The Economet- rics of Financial Markets, Princeton: Princeton University Press, 1997.

CHIANG, T. C.; DOONG, S. C.. Empirical Analysis of Stock Returns and Volatility: Evidence from Seven Asian Stock Markets Based on TAR-GARCH Model. Review of Quantitative Finance and Accounting, n. 17 p. 301-318, 2001.

COCHRANE, D.; ORCUTT, G. H.. Application of Least Squares Regression to Relationships Containing Autocorrelated Error Terms. Journal of the American Statistical Association, n. 44, p. 32-61, 1949.

DICKEY, D. A.; FULLER, W. A.. Distribution of Estimators for Time Series Regressions with a Unit Root. Journal of the American Statistical Association, n. 74, p. 427-31, 1979.

DURBIN, J.; WATSON, G. S.. Testing for Serial Correlation in Least Squares Regression. Biometrika, n. 38, p. 159-71, 1951.

ECONOMÁTICA. Banco de Dados: Cotações das ações e Ibovespa. Data base: fevereiro de 2003.

ENGLE, R. F.. Autoregressive Conditional Heteroskedastic- 
ity with Estimates of the Variance of United Kingdom Inflation. Econometrica, n. 50 (4), p. 987-1007, 1982.

FAMA, E.. Market efficiency, long-term returns, and behavioral finance. Journal of Financial Economics, n. 49, p. 283-306, 1998.

GERARD, B.; NANDA, V.. Trading and manipulation around Seasoned Equity Offerings. Journal of Finance, vol. XLVIII, n. 1, March 1993.

HERTZEL, M.; LEMMON, M.; LINCK, J. S.; REES, L.. Long-run Performance Following Private Placements of Equity. Journal of Finance, vol. 57, n. 6, Dec. 2002.

HESS, A. C.; FROST, P. A.. Tests for price effects of new issues of seasoned securities. Journal of Finance, vol. XXXVI, $n$. 1, March 1982

JENSEN, M. C.. Agency Costs of Free Cash Flow, Corporate Finance and Takeovers. Harvard Business Review, n. 1984, p. 109$121,1986$.

JENSEN, M. C.; MECKLING, W. H.. Theory of the Firm: Managerial Behavior, Agency Costs and Ownership Structure. Journal of Financial Economics 3, p. 305-360, 1976.

KADYIALA, P.; RAU, P. R.. Investor reaction to corporate event announcements: under-reaction or over-reaction? Working paper, Purdue University. Journal of Business, June 2001.

KOTHARI, S. P.; ZIMMERMAN, J.. Price and Return Models. Journal of Accounting and Economics n. 20, p. 155-192, 1995.

LEAL, R. P. C.; AMARAL, A. S.. Um Momento para o Insider Trading: O Período Anterior ao Anúncio de uma Emissão Pública de Ações. Em LEAL, R. P. C., COSTA Jr., N. C. A. e LEMGRUBER, E. F. (Org.), Finanças Corporativas, Coleção COPPEAD de Administração, São Paulo: Atlas, 2000.

LOUGHRAN, T.; RITTER, J.. The New Issues Puzzle, Journal of Finance, n. 50, p. 23-51, Mar. 1995.
The Operating Performance of Firms Conducting Seasoned Equity Offerings, Journal of Finance, n. 52, p. 18231850, 1997.

LUCAS, D. J.; MCDONALD, R. L.. Equity Issues and Stock Price Dynamics. Journal of Finance, n. 45, September 1990.

MACKINLAY, A. C.. Event studies in economics and finance. Journal of Economic Literature, vol. 35, issue 1, p. 13-39, Mar. 1997.

MIKKELSON, W. H.; PARTCH, M. M.. Valuation Effects of Security Offerings and the Issuance Process. Journal of Financial Economics, n. 15, 1986.

MILLER, M. H.; ROCK, K.. Dividend Policy under Asymmetric Information. Journal of Finance, n. 40, p 1031-1051, 1985.

MYERS, S. C.; MAJLUF, N. S.. Corporate Financing and Investment Decisions When Firms Have Information that Investors Do Not Have, Journal of Financial Economics, n. 13, p. 187-221, 1984.

SCHOLES, M.. The Market for Securities: Substitution versus Price Pressure and the Effects of Information on Stock Prices, Journal of Business, n. 45, April 1972.

SOARES, R. O.; ROSTAGNO, L. M.; SOARES, K. T.. Estudo de Evento: O Método e as Formas de Cálculo do Retorno Anormal, XXVI ENANPAD, Anais do ENANPAD, Salvador (BA), 2002.

SPIESS, D. K.; AFFLECK-GRAVES, J.. Under-performance in long-run stock returns following seasoned equity offerings. Journal of Financial Economics, n. 38, p. 243-267, 1995.

WHITE, H.. A Heteroskedasticity-Consistent Covariance Matrix Estimator and a Direct Test for Heteroskedasticity, Econometrica, n. 48, p. $817-38,1980$.

\section{Endereço dos autores:}

Universidade de Brasília

Faculdade de Estudos Sociais Aplicados

Asa Norte, S/N

Brasília - DF

70910-900
Universidade Católica de Brasília

QS 07, Lote 01 - EPCT - Águas Claras

Taguatinga - DF

72030-170

R. Cont. Fin. - USP, São Paulo, n. 40, p. 25 - 36, Jan./Abr. 2006 


\section{ANEXO: EMISSÕES DA AMOSTRA}

\begin{tabular}{|c|c|c|c|c|c|}
\hline $\mathbf{N}^{\circ}$ & EMPRESA & $\begin{array}{l}\text { DATA DO } \\
\text { ANÚNCIO }\end{array}$ & $\mathbf{N}^{\circ}$ & EMPRESA & $\begin{array}{l}\text { DATA DO } \\
\text { ANÚNCIO }\end{array}$ \\
\hline 1 & Acesita PN & 05/26/1995 & 41 & Embraer PN & 04/19/1995 \\
\hline 2 & & 06/26/1996 & 42 & & 04/19/1996 \\
\hline 3 & & 10/22/1999 & 43 & F Cataguazes PNA & 03/11/1996 \\
\hline 4 & Adubos Trevo PN & 11/14/1996 & 44 & Gerdau PN & 02/18/1994 \\
\hline 5 & Ambev PN & 08/27/1993 & 45 & Inepar Construcoes PN & 09/23/1993 \\
\hline 6 & Antarct Nordeste PNA & 06/01/1998 & 46 & Ipiranga Pet PN & $10 / 25 / 1993$ \\
\hline 7 & Banrisul PN & 07/30/1993 & 47 & Itausa PN & $01 / 21 / 2000$ \\
\hline 8 & Belgo Mineira PN & 03/18/1998 & 48 & & 03/12/2001 \\
\hline 9 & Bradesco PN & 05/11/1993 & 49 & Itautec ON & 09/06/1995 \\
\hline 10 & & $12 / 27 / 1993$ & 50 & & $12 / 15 / 1998$ \\
\hline 11 & & 05/19/1994 & 51 & Kuala PN & 01/19/1996 \\
\hline 12 & & 05/26/1995 & 52 & Light ON & 04/12/1999 \\
\hline 13 & & 12/04/1995 & 53 & & 04/29/2002 \\
\hline 14 & & 12/10/1997 & 54 & Marcopolo PN & 09/27/2002 \\
\hline 15 & & 12/10/1998 & 55 & Minupar PN & 09/16/1994 \\
\hline 16 & & 12/08/1999 & 56 & Net PN & $10 / 07 / 1999$ \\
\hline 17 & & $12 / 08 / 2000$ & 57 & Nordon Met ON & 03/20/1997 \\
\hline 18 & & $12 / 26 / 2002$ & 58 & Pao de Acucar PN & 05/05/1997 \\
\hline 19 & Bradespar PN & $11 / 24 / 2000$ & 59 & Sabesp ON & $11 / 16 / 1999$ \\
\hline 20 & Brasil ON & 12/30/1992 & 60 & & $12 / 23 / 1999$ \\
\hline 21 & & 09/21/1993 & 61 & Sudameris ON & 07/07/1994 \\
\hline 22 & Brasil T Par ON & 02/09/2001 & 62 & & 02/28/1997 \\
\hline 23 & & 03/27/2002 & 63 & & 07/22/1998 \\
\hline 24 & Brasil Telecom PN & 04/25/1994 & 64 & Telasa Celular BN & 03/01/2001 \\
\hline 25 & & 03/29/2001 & 65 & Tele Celular Sul PN & 03/19/2001 \\
\hline 26 & Bunge Brasil PN & 09/14/1995 & 66 & Tele Leste Celular PN & $02 / 23 / 2000$ \\
\hline 27 & Celesc PNB & 09/19/1996 & 67 & Tele Nordeste Celul PN & 03/19/2001 \\
\hline 28 & Cesp PN & 09/26/1994 & 68 & & 03/04/2002 \\
\hline 29 & Chapeco PN & $10 / 19 / 1993$ & 69 & Tele Sudeste Celula PN & $02 / 22 / 2002$ \\
\hline 30 & & $11 / 23 / 1995$ & 70 & Telebras Remanescen PN & $01 / 02 / 2001$ \\
\hline 31 & & 08/31/1999 & 71 & Telemar PN & $04 / 19 / 2000$ \\
\hline 32 & & 11/11/1999 & 72 & & $04 / 25 / 2002$ \\
\hline
\end{tabular}


Continuação

\begin{tabular}{|l|l|c|c|c|l|c|}
\hline No & \multicolumn{1}{|c|}{ EMPRESA } & $\begin{array}{c}\text { DATA DO } \\
\text { ANÚNCIO }\end{array}$ & No $^{-1}$ & EMPRESA & $\begin{array}{c}\text { DATA DO } \\
\text { ANÚNCIO }\end{array}$ \\
\hline 33 & & $08 / 03 / 2000$ & & 73 & Telemig Celul Part PN & $04 / 12 / 2001$ \\
\hline 34 & & $09 / 27 / 2000$ & & 74 & Telesp Operac PN & $04 / 07 / 1994$ \\
\hline 35 & Comgas PNA & $04 / 27 / 2001$ & & 75 & & $10 / 11 / 1994$ \\
\hline 36 & Copel PNB & $07 / 22 / 1997$ & & 76 & & $04 / 12 / 1995$ \\
\hline 37 & Coteminas PN & $03 / 08 / 1994$ & & 77 & & $04 / 25 / 1996$ \\
\hline 38 & CRT Celular PNA & $06 / 07 / 1999$ & & 78 & & $10 / 02 / 1997$ \\
\hline 39 & Duratex PN & $09 / 08 / 2000$ & & 79 & Tractebel ON & $08 / 09 / 2000$ \\
\hline 40 & Eluma PN & $11 / 20 / 1995$ & & 80 & Votorantim C P PN & $11 / 01 / 1993$ \\
\hline
\end{tabular}

